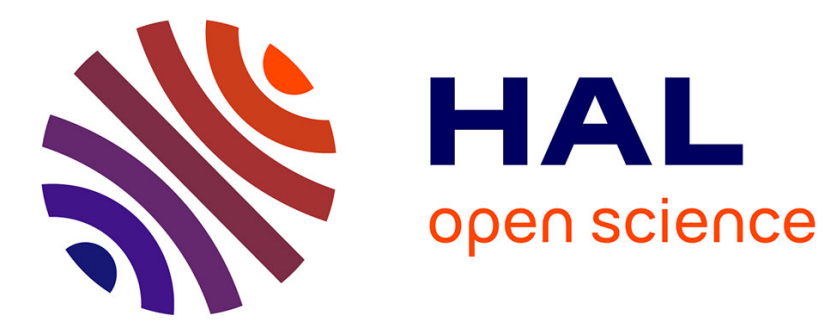

\title{
Parler de management et de gestion éthique. Un exemple : le recueil des directives anticipées
}

Karine Bréhaux, Corinne Frémond-Lejeune

\section{To cite this version:}

Karine Bréhaux, Corinne Frémond-Lejeune. Parler de management et de gestion éthique. Un exemple : le recueil des directives anticipées. Revue management \& avenir, 2015, 82 (8), pp.57. 10.3917/mav.082.0057 . hal-03006588

\section{HAL Id: hal-03006588 \\ https://hal.science/hal-03006588}

Submitted on 1 Dec 2020

HAL is a multi-disciplinary open access archive for the deposit and dissemination of scientific research documents, whether they are published or not. The documents may come from teaching and research institutions in France or abroad, or from public or private research centers.
L'archive ouverte pluridisciplinaire HAL, est destinée au dépôt et à la diffusion de documents scientifiques de niveau recherche, publiés ou non, émanant des établissements d'enseignement et de recherche français ou étrangers, des laboratoires publics ou privés. 
PARLER DE MANAGEMENT ET DE GESTION ÉTHIQUE. UN EXEMPLE : LE RECUEIL DES DIRECTIVES ANTICIPÉES

\author{
Karine Bréhaux, Corinne Frémond-Lejeune
}

Management Prospective Ed. | « Management \& Avenir »

2015/8 Nº 82 | pages 57 à 80

ISSN 1768-5958

Article disponible en ligne à l'adresse :

https://www.cairn.info/revue-management-et-avenir-2015-8-page-57.htm

Distribution électronique Cairn.info pour Management Prospective Ed..

(C) Management Prospective Ed.. Tous droits réservés pour tous pays.

La reproduction ou représentation de cet article, notamment par photocopie, n'est autorisée que dans les limites des conditions générales d'utilisation du site ou, le cas échéant, des conditions générales de la licence souscrite par votre établissement. Toute autre reproduction ou représentation, en tout ou partie, sous quelque forme et de quelque manière que ce soit, est interdite sauf accord préalable et écrit de l'éditeur, en dehors des cas prévus par la législation en vigueur en France. Il est précisé que son stockage dans une base de données est également interdit. 


\title{
Parler de management et de gestion éthique. Un exemple: le recueil des directives anticipées
}

\author{
Karine BRÉHAUX ${ }^{1}$ \\ Corinne FRÉMOND-LEJEUNE ${ }^{2}$
}

Résumé

Les directives anticipées, en référence à la loi de 2005, dite Loi de Léonetti, constituent un dispositif d'expression, par anticipation, de la volonté du patient quant à sa fin de vie, pour le cas où il ne serait plus en capacité de le faire.

Une étude a été menée dans un centre hospitalier sur la phase de recueil. Comme toute activité, ce processus est soumis à des dangers. Face à eux ou en situation dangereuse, la méthode d'Analyse Globale des Risques (AGR), appliquée au sujet, permet d'identifier et de caractériser les scénarios conduisant à des évènements redoutés afin de définir des actions de réduction des risques assorties ou non de paramètres de sécurisation. Elles sont soumises à validation des dirigeants sur la base des risques majeurs et d'un bilan financier. Le coordonnateur de la gestion des risques associés aux soins de l'établissement conduit la démarche et anime le groupe d'experts du système étudié.

Cette étude a permis d'identifier 332 scénarios dont 42,77 \% associés à un risque tolérable sous contrôle et $11,14 \%$ à un risque non acceptable, définis ainsi par la matrice de criticité, outil managérial de décision. Parmi ces derniers, trois risques majeurs ont été identifiés sur la base des valeurs maximales des risques moyens. Deux d'entre eux étaient associés à l'étape d'évaluation des capacités du patient à exprimer ses volontés et le troisième était associé à l'étape de recueil de la décision du patient de rédiger ou non ses directives anticipées. Deux actions de réduction des risques, de nature préventive et économiquement rentables, chacune consolidée par un paramètre de sécurité ont été retenues en guise de traitement.

1 Karine BRÉHAUX: Docteur de l'IEP de Paris, Reims.

2 Corinne FRÉMOND-LEJEUNE: Praticien hospitalier chef de pôle, Coordonnateur de la Gestion des Risques Associés Aux Soins, Troyes. 
L'élaboration d'un projet managérial énonce des valeurs partagées et cette étude permet de les décliner objectivement: rendre compatible les évolutions de l'hôpital avec la démarche éthique, rappeler les valeurs communes du service public hospitalier en les corrélant avec les droits des personnes et les valeurs éthiques (égalité, neutralité, médiation, respect de la réglementation, respect du consentement de la personne). Cela nécessite de développer des principes de management éthique participatif où de nouveaux profils de managers (à la fois directeur d'établissement et éthicien?) seraient appréciés et où le coordonnateur de la gestion des risques associés aux soins de l'établissement pourrait être un nouveau manager créatif.

\section{Abstract}

The anticipated directives, in references to the LEONETTI's law promulgated in 2005 , are a way to express patients' choices concerning their end of life in cases where they would not be able to do so. A study was conducted in a hospital on the collection phase.

As an activity, this process is subject to dangers. In this situation, the method of Global Risk Analysis, applied to the subject, allows the scenarios leading to feared events to be identified and characterised. Actions to reduce the risks and securing parameters can be defined and submitted to the leaders in order to consult major risks data bases and financial statements.

The risk management coordinator leads the process and guides the expert specialised in the studied system. This study allows 332 scenarios to be identified with $42.77 \%$ matching tolerable risks under control and $11.14 \%$ matching unacceptable risks regarding the criticality matrix. As part of them, three major risks were identified considering the maximal values of the average risks. Two of them were associated to the evaluating stage of patients' capacity to express their will and the third one was associated to the collecting stage of patients' choice to write or not their anticipated directives. Two actions, preventive, financially interesting and strengthened by secure parameters were chosen to reduce the risks. The development of a managerial project sets out shared values and this study allows to decline objectively: make it compatible developments of the hospital with the ethical approach, remember the common values of the public hospital service by correlating them with the rights of persons and ethical values (equality, neutrality, mediation, compliance, respect of the person's consent.). This requires the development of participatory ethical management principles which new profiles managers (both warden and ethicist?) Would be appreciated and where the coordinator of risk management associated with the care of the institution could be a new creative manager. 


\section{Parler de management et de gestion éthique. Un exemple : le recueil des directives anticipées}

\section{Introduction}

Selon le Larousse, une directive est « une indication générale donnée par l'autorité politique, administrative...Décision par laquelle l'administration détermine à l'avance la manière dont elle usera de son pouvoir discrétionnaire, c'est une instruction générale émanant du haut commandement et exprimant sa pensée ou son idée de manœuvre». Anticipée : «Agir comme si on pouvait disposer de quelque chose qui n'existe pas encore ». Parler de « directives anticipées » c'est confronter une logique d'action (le sujet décide de) avec une logique de contrainte d'ordre politique. D'emblée, nous sommes dans un registre réglementaire, pour rappel l'article 1111-11 du code de la santé publique: «Toute personne majeure peut rédiger des directives anticipées pour le cas où elle serait un jour hors d'état d'exprimer sa volonté. Ces directives anticipées indiquent les souhaits de la personne relatifs à sa fin de vie concernant les conditions de la limitation ou l'arrêt de traitement. Elles sont révocables à tout moment» (Code de la Santé Publique en vigueur). Entre la directive administrative, l'indication politique contraignante et la directive anticipée, il y a actuellement un saut car la directive anticipée est personnelle et non obligatoire. Le registre réglementaire octroie une possibilité (liberté ?) de directive anticipée sans contrainte («non opposable») quant à la réalisation du contenu par les soignants. Dans le débat actuel autour de la fin de vie, le CCNE propose de créer deux types de directives anticipées :

- Premier type : «déclarations anticipées de volonté», que toute personne, malade ou pas, songeant à sa fin de vie serait invitée à rédiger. Elles seraient destinées à l'inciter, non pas à anticiper sa mort, mais plutôt à réfléchir à sa propre fin de vie : pourraient y être exposés ses souhaits en termes de lieu de vie ou de mode de prise en charge.

- Deuxième type : il répond à la situation d'une personne atteinte d'une maladie grave ou potentiellement létale. La valeur intrinsèque des « directives anticipées » est différente: elles constituent un véritable outil de dialogue avec le malade. Il est alors essentiel pour leur rédaction, qu'un accompagnement par un professionnel de santé soit proposé à la personne intéressée, afin d'aborder ce temps très délicat avec tact et mesure et d'aider à l'élaboration du document dans le but de garantir son effectivité. Ces «directives anticipées » permettraient d'anticiper les décisions qui doivent être prises, compte-tenu de l'évolution de la maladie et des différentes options qui s'ouvriront. Leur intérêt, à la fois pour le patient et le médecin, est ainsi manifeste dans certaines maladies chroniques ou dégénératives (Aubry, 2014).

Mais avant de discuter autour des deux types de directives anticipées et de tenter de formaliser les pratiques managériales et soignantes, revenons à la question première du CCNE : «Quelle est la valeur de directives anticipées alors que la personne, ayant certes toutes ses capacités, n'est pas entrée ou entre à peine dans la maladie?». Pour cela nous vous proposons un détour par la philosophie. L'idée ici défendue est que la formalisation de directives anticipées, par une personne, à un instant donné de sa propre vie (certes, peut-être pas représentatif de l'ensemble de sa vie, si courte soit-elle) réactive la question philosophique de l'union de l'âme et du corps et d'une re-fondation de la conception de la personne non scindée sur une séparation stricte âme-corps, voir une instrumentalisation de l'un par l'autre (controverses philosophiques autour de 
l'exploitation des parties de son corps humain à son propre compte, voir les travaux de Robert Nozick, penseur libertarien, qui tient ce type de raisonnement : «Si je souhaite vendre mon rein afin de gagner de l'argent c'est mon droit!» (Nozick, 1975) mais sur une unité commune de l'âme et du corps. Ici est observé une résurgence de la position spinoziste de l'âme et du corps, deux entités, unies mais se dévoilant sur deux modes de réalités distincts : substance étendue, substance pensante. Au-delà des questions bioéthiques relatives à la personne, la réglementation en vigueur impose un changement des pratiques soignantes dans son appréhension de ce consentement écrit de la personne hospitalisée. Changements qui ont pour conséquence une reconfiguration des aspects managériaux et budgétaires afin de faire face à ces prises de considérations éthiques de la volonté écrite du patient. Nous défendrons ici, à travers ce cas, une vision managériale nouvelle car fondée sur des concepts éthiques. Ce mouvement d'éthicisation d'une logique managériale au sein des établissements sanitaires impose l'apparition de nouveaux paramètres dans les prises de décisions et dans le profil des acteurs : compétences en éthique, management reposant sur des concepts écrits, conciliation des logiques réglementaires, gestionnaires et comptables.

\section{1. «Les directives anticipées : de l'origine à la formalisation des pratiques »}

\subsection{Les directives anticipées: le renvoi à une conception personnelle de la vie que je souhaite mener... jusqu'au bout.}

\subsubsection{L'indication des souhaits : l'expression individuelle, le souhait d'une personne. Identification de la volonté individuelle (Moi) au corps souffrant}

L'indication des souhaits qu'une personne se trouvant dans un état d'inconscience, concerne son corps et l'atteinte consentie qu'il puisse y être fait. De prime abord, nous sommes confrontés au principe d'intégrité physique (articles 16 et 16-1 du Code civil français) et du consentement de la personne à autoriser qu'il soit fait tel ou tel traitement ou a contrario le cas d'une abstention thérapeutique. D’entrée de jeu, on opère une séparation entre le corps et la volonté individuelle, et somme toute renonçons à l'héritage traditionnel philosophique d'une consubstancialité du corps et de l'âme. Faisons-nous fausse route? L'indication des souhaits est formulée sur une perception d'une souffrance à venir, d'un supplice futur à endurer. Ce que le sujet recherche c'est l'absence de souffrance (l'ataraxie), le refus du supplice, ce que Michel Foucault dans Surveiller et punir, rappelle par la définition du supplicié qui dans les siècles passés était qualifié de «patient» celui qui souffre. En me projetant sur ce futur incertain, j'essaie de mettre en place des mesures qui empêchent mon corps de souffrir. L'identification entre la volonté individuelle (ses actions) et le corps-objet de souffrance est totale: "L'homme dont on nous parle et qu'on invite à libérer est déjà lui-même l'effet d'un assujettissement bien plus profond que lui. Une 'âme' l'habite et le porte à l'existence, qui est elle-même une pièce dans la maîtrise que le pouvoir exerce sur le corps. L'âme, effet et instrument d'une anatomie politique ; l'âme, prison du corps » (Foucault, 1975). 


\section{Parler de management et de gestion éthique. Un exemple : le recueil des directives anticipées}

Je refuse que mon corps souffre et souhaite mettre en place un ensemble de mesures pour éviter cette situation.

\subsubsection{Le corps est-il l'égal de l'âme?}

Parler de valeur, c'est pour le coup opérer un jugement entre deux éléments et « évaluer » lequel des deux est premier sur l'autre. La question philosophique du rapport de force entre l'âme et le corps apparaît: de la conception scholastique de Maître Eckhart qui voit le corps comme le signifié de l'âme (Maître Eckhart, 1975) à la vision habermasienne du refus de réification de la personne humaine, les courants philosophiques ont montré les différents rapports possibles entre les deux entités. Poser des indications sur ce que j'aimerais qu'il soit fait à mon corps dans un contexte de fin de vie, c'est reconnaître alors une prédominance du moi sur le corps appartenant à l'ordre des choses. L'idée qu'un corps sans cerveau a moins de sens, reste une vision partagée dans des controverses autour du transhumanisme, de l'interface cerveau-machine, sur les possibilités via les sciences de développer notre potentiel d'essence humaine malgré nos corps inachevés. Le corps semble moins parfait que l'âme, ce que résume Descartes : «Je suis une chose qui pense, c'est-à-dire qui doute, qui en ignore beaucoup, qui aime, qui hait, qui veut, qui ne veut pas, qui imagine et qui sent». Pour Descartes, le sujet dans ses dispositions spirituelles et naturelles reste imparfait, seul Dieu est parfait et reste l'auteur de toute chose et être : «Par le nom de Dieu, j'entends une substance infinie, éternelle, immuable, indépendante, toute connaissante, toute-puissante, et par laquelle moi-même, et toutes les autres choses qui sont (s'il est vrai qu'il y en ait qui existent) ont été crées» (Descartes, 2005). Dieu en tant que tiers détermine l'évolution de mon être, si bien que je ne peux pas prédéterminer des événements à venir et penser que le cours de mon existence et la conception de ma propre existence n'est pas susceptible de changer : «Car tout le temps de ma vie peut être divisé en une infinité de parties, chacune desquelles ne dépend en aucune façon des autres; et ainsi, ce n'est qu'en ce moment quelque cause me produise et me crée, pour ainsi dire, derechef, c'est-dire me conserve " (Descartes, 2005). Le fait que je ne puisse pas prévoir l'évolution de mon moi, de mon corps, pour des raisons multiples et des facteurs externes ou internes (intervention du tiers - la figure divine - chez Descartes et Maître Eckhart, chez Nietzsche (Nietzsche, 2000), le «surhomme» le refus de l'aliénation de la raison et le passage par la défiguration...) nous renvoie à une signification : la capacité autoréflexive de l'individu, la prise de conscience de sa finitude.

\section{Les directives anticipées : de l'origine à la formalisation des pratiques}

Dans la pratique soignante au quotidien, cette question de déclaration et donc de rédaction des directives anticipées par les usagers vers les soignants est délicate, voire tabou car touchant à la finitude humaine des uns et des autres. Le dispositif actuel semble imparfait selon les demandes sociétales en présence. Le facteur temps est important dans la prise en charge des personnes souffrantes, il reste primordial de " prendre le temps ", car cette question est associée inévitablement à la souffrance, à la perte d'autonomie. Dans notre société française actuelle, la rédaction des directions 
anticipées est encore peu formalisée, peu de personnes y recourent selon les récents sondages. La décision appartient au médecin, relève de sa responsabilité professionnelle et pénale (Code de déontologie médicale en vigueur). La loi Léonetti aboutit à un compromis entre les droits des patients et les devoirs des soignants, s'inscrivant dans la perspective de la loi du 4 mars 2002 relative aux droits des patients. Cette loi pose le cadre d'une relation qui se construit: principe d'autonomie, paternalisme médical associé au principe de bienveillance, consentement du patient et devoirs des soignants permettant l'échange entre la personne en souffrance, les soignants et l'entourage. À plusieurs, on réfléchit mieux! La dynamique entre les protagonistes est volontairement lente car laissant du temps au temps, aux échanges entre les personnes, à la consultation des directives anticipées par l'équipe soignante, au rôle que joue la personne de confiance quand malheureusement la personne malade ne peut plus communiquer son avis aux soignants et non soignants. La responsabilité est partagée, la parole délivrée. Socialement, on observe un désintéressement global des connaissances du dispositif du déni. Est-ce que le dispositif actuel est adéquat? Pour qui? Par rapport aux vrais besoins des patients aujourd'hui? Quelles sont les demandes des personnes? Est-ce que la perte de confiance globale dans le système de santé ne fragilise pas le temps de la fin qui est si délicat? La question de reconnaître ou pas une valeur juridique aux directives anticipées n'est peut-être pas la bonne question, au sens éthique du terme. La perspective de figer une vision des conditions de sa propre mort et de la fin de sa vie à un moment donné de sa vie, risque -en dépit d'une possibilité certes de les modifier et d'une durée pour - de mettre la personne dans une situation unique. La singularité de la personne, dans ses aspirations, choix, sa liberté d'être, se trouve réduite à quelques lignes écrites sur un papier blanc. Ainsi nous renouvelons le débat actuel «j'ai un corps ou je suis un corps? » sous l'angle du corps comme unique représentant de la personne. La certification, introduite au sein du système de santé par l'ordonnance numéro 96-346 du 24 avril 1996 portant sur la réforme hospitalière, est la procédure d'évaluation externe des établissements de santé publics et privés effectuée par des professionnels mandatés par La Haute Autorité de Santé. Indépendante de l'établissement et des organismes de tutelle, elle consiste en une analyse du fonctionnement global de l'établissement et non de chaque secteur d'activité même si chaque type de prise en charge du patient est analysé. Concernant la quatrième procédure, on parle de management par la qualité et non plus de management de la qualité. La fin de vie y est abordée comme un processus, analysé selon le principe de la roue de Deming : prévoir, mettre en œuvre, évaluer et améliorer. Parmi les attendus énoncés, on retrouve : «Des modalités de recueil de la volonté du patient et, le cas échéant, de conservation des directives anticipées sont définies » et « les patients adultes reçoivent des informations écrites sur leur droit d'accepter ou de refuser des traitements de maintien en vie ou les traitements de réanimation et sur leur droit d'établir des directives anticipées ». Ces obligations réglementaires imposent à leur tour une réflexion pour la direction d'un établissement sanitaire et sa traduction en termes de gestion budgétaire et de management. Quels choix comptables sont opérants? Quel type de management mettre en place afin d'atteindre ces objectifs qualitatifs? 


\section{Parler de management et de gestion éthique. Un exemple : le recueil des directives anticipées \\ 3. Parler de management et de gestion éthique. Un cas : le recueil des directives anticipées}

En 2013, et dans le cadre de sa thèse de mastère spécialisé en gestion des risques des établissements et réseaux de santé de l'Ecole Centrale Paris, le Dr. Corinne FrémondLejeune a mené une étude intitulée «Application de la loi Léonetti relative aux droits des patients en fin de vie: Cartographie et gestion des risques du recueil des directives anticipées dans la phase d'encouragement des patients à les rédiger » (Fremond-Lejeune, 2012-2013).

Elle met alors en exergue une initiative locale innovante d'amélioration des pratiques basée sur une analyse de risques autour du recueil des directives anticipées.

Cette étude a été menée dans un centre hospitalier en pleine réflexion sur le sujet. En effet, la visite prochaine de certification V2014 et la démarche engagée pour obtenir des lits identifiés soins palliatifs sur le pôle de Soins de Suite et de Réadaptation avaient fait naître un débat qui s'inscrivait complètement dans la réflexion nationale.

Trois enquêtes préliminaires à l'étude ont été réalisées en juillet 2013. Elles ont révélé la méconnaissance du dispositif par les soignants. Même constat chez les patients interrogeables, qui, par ailleurs, se montraient globalement favorables à être accompagnés en vue de prendre leur décision et/ou de rédiger leurs directives anticipées.

Toutes les structures internes au pôle ont été incluses dans l'étude : Soins de Suite Gériatriques, Polyvalents, Locomoteurs et Addictologie.

De même, ont été considérés tous les patients admis, quels que soient leur lieu d'origine, leur motif d'hospitalisation, leur âge et en considérant d'emblée qu'ils n'avaient pas rédigé leurs directives anticipées au préalable. Ce dernier critère se justifiait au regard des chiffres nationaux qui montraient que seulement 2,5\% des patients concernés par les directives anticipées les avaient rédigées au préalable et les résultats des enquêtes internes de 2013 qui montraient qu'un seul patient disposait de ses directives anticipées.

Enfin, seule la phase d'encouragement des patients a été étudiée en raison du temps imparti et considérant qu'elle constituait la première étape du recueil des directives anticipées. Par encouragement, il faut entendre les moyens mis en œuvre pour permettre au patient de prendre sa décision de rédiger ou non ses directives anticipées et non une incitation à le faire. 


\subsection{La méthode appliquée au sujet: 1'Analyse Globale des Risques (AGR) comme levier à la mise en place d'un " management éthique " ${ }^{3}$}

Le traitement du sujet sous l'angle «sécurité de système » n'est pas apparu comme une évidence pour les professionnels concernés. Ils méconnaissaient les concepts de la gestion des risques, l'existence d'évènements indésirables dans ce domaine et restaient méfiants à l'égard de résultats issus d'une approche dite rationnelle en opposition à un domaine où tout leur semblait être de l'ordre de l'analyse subjective de cas particuliers.

Une séance introductive à la notion de gestion des risques a donc été nécessaire.

La méthode d'Analyse Globale des Risques (AGR) a ensuite été présentée par le Coordonnateur de la Gestion des Risques Associés aux Soins (CGRAS) de l'établissement puis retenue sur les arguments suivants :

- Le traitement du thème sous l'angle sécurité de système était innovant et complémentaire aux approches actuelles ;

- $\quad$ La méthode repose au préalable sur un système (ou processus) préalablement défini. Une activité comme le recueil des directives anticipées est un système ;

- $\quad$ Comme toute activité, le recueil des directives anticipées est soumis à des dangers. Face à eux ou en situation dangereuse, la méthode permet d'identifier et de caractériser les scénarios conduisant à des évènements redoutés afin de définir des actions de réduction des risques et de sécurisation ultérieure ;

- L'effort de sécurité est adapté aux objectifs visés. L'objectif étant ici de permettre à tout patient admis et en capacité de s'exprimer de pouvoir se prononcer sur sa volonté de rédiger ou non ses directives anticipées ;

- $\quad$ Connue et utilisée depuis de nombreuses années dans les activités industrielles, elle est maintenant fréquemment appliquée à la gestion des risques des établissements de santé, pour une activité nouvelle ou déjà existante ;

- $\quad$ Elle se pratique avec les professionnels de terrain, experts du système étudié, garant de la pertinence de l'analyse et sous la conduite d'un expert métier en gestion des risques, garant de la méthode.

Ainsi, au regard du thème traité et du contexte local, l'AGR s'est présentée comme une méthode innovante, pertinente, applicable et adaptée.

À l'origine, il était prévu de pouvoir comparer l'application de la loi de 2005, versus la loi révisée de 2013, et notamment de savoir si les nouvelles dispositions allaient supprimer tout ou partie des risques identifiés ou en générer de nouveaux. En l'absence

3 L'éthique regroupe un ensemble de règles qui se différencient des règles juridiques et les complètent. Elle a pour objet d'apporter la réponse la plus appropriée aux problèmes liés aux caractères particuliers des situations et vise à répondre à la question «comment agir au mieux?».

Le plan d'actions à mettre en œuvre repose sur la déclinaison des objectifs suivants: 1'élaboration d'un projet managérial, la volonté de créer un véritable lieu d'échanges à destination des professionnels et du grand public, la promotion des formations. 


\section{Parler de management et de gestion éthique. Un exemple : le recueil des directives anticipées}

de révision de la loi, le travail a donc été réajusté. L'analyse des risques a bien été réalisée a priori, puisque rien n'était mis en place au niveau du pôle, mais au regard de l'application de la loi actuelle.

\subsubsection{La méthode: 1'Analyse Globale des Risques en bref}

L'Analyse Globale des Risques est une méthode d'analyse des risques, inductive et semi-quantitative.

En termes de méthodologie, elle se déroule en trois temps:

- L'analyse système dite AGR système ;

- L'analyse scénarios dite AGR scénarios ;

- L'identification des risques majeurs et l'élaboration du plan d'actions de réduction des risques assorti du catalogue des paramètres de sécurité.

L'AGR système comprend elle-même trois étapes :

- La description du système étudié :

La démarche utilisée est celle de l'analyse fonctionnelle. Elle permet de limiter l'oubli d'exigences et favorise l'innovation en évitant de proposer directement des solutions ou de s'enfermer dans celles déjà existantes.

Elle se déroule en deux temps :

- L'analyse fonctionnelle externe exprimant le juste besoin et la recherche des fonctions attendues et leurs caractéristiques du point de vue de l'utilisateur du système. Elle est conduite selon la méthode APTE dite également méthode de la bête à cornes ou taureau système. Le système est ensuite situé dans son environnement en recherchant les éléments extérieurs qui interagissent avec lui et qu'il convient de prendre en compte dans la démarche d'analyse des risques.

- L'analyse fonctionnelle interne permettant de décomposer les fonctions principales du système étudié en phases et sous phases et d'en identifier les performances et contraintes. Elle est conduite selon la méthode RELIASEP qui décompose ces fonctions principales selon un principe : je capte, je transforme et je transmets.

- L'élaboration de la cartographie des dangers :

Elle correspond à une liste structurée de dangers auxquels le système est exposé. Ils sont identifiés à partir d'une liste de dangers génériques adaptés au monde de la santé (grandes catégories de domaines dans lesquels les dangers sont susceptibles d'apparaître), elle-même déclinée en une liste de dangers, d'éléments ou d'événements dangereux spécifiques au système étudié.

- L'élaboration de la cartographie des situations dangereuses :

Elle résulte de l'évaluation des interactions «dangers/phases du système ». Chacune d'entre elles est codée et donne lieu à analyse ou non dans le cadre de l'AGR scénarios selon l'importance de l'interaction décelée. Les interactions fortes à très fortes nécessitent alors une analyse immédiate dans le cadre de l'AGR scénarios.

L'AGR scénarios comprend trois étapes :

- L'élaboration des paramètres d'évaluation:

Est considérée la gravité des conséquences (G) de chaque scénario d'événement redouté identifié. Cette gravité est estimée, a priori, du point de vue des performances à atteindre par le système étudié et du point de vue de la sécurité. Une échelle de gravité est construite en amont de l'analyse des scénarios, soumise à l'avis des dirigeants. 
Est ensuite considérée la vraisemblance de la gravité des conséquences (V) de chaque scénario d'événement redouté identifié. Une échelle de vraisemblance est construite en amont de l'analyse des scénarios, soumise à l'avis des dirigeants.

Est enfin considéré le risque moyen assorti à chaque scénario et correspondant au produit de G et V.

- L'élaboration des éléments d'acceptation du risque et de décision :

La criticité du risque est le résultat de la fonction de décision qui dépend des deux paramètres d'évaluation: $G$ et $V$. Un tableau de criticité (ou référentiel d'acceptabilité), en référence à une échelle du même nom, permet de qualifier le risque comme acceptable, tolérable sous contrôle ou non acceptable et détermine l'obligation ou non d'entreprendre des actions. Cette matrice est élaborée en amont de l'analyse des scénarios, soumise à validation des dirigeants.

Sont également estimés les pertes financières en l'absence de traitement des risques initiaux et les efforts financiers liés à leurs traitements.

- L'élaboration de la cartographie des risques:

L'analyse des scénarios issus de chaque situation dangereuse s'opère au moyen des échelles de gravité et vraisemblance préalablement définies. La matrice de criticité est appliquée permettant de qualifier le risque. Les scénarios dont le risque initial est non acceptable ou tolérable sous contrôle font l'objet d'identification d'actions de réduction des risques agissant sur G et/ou V. Elles se complètent de paramètre de sécurité lorsque le risque résiduel n'est pas acceptable. Le coût du risque et de son traitement sont appréciés.

L'identification des risques majeurs et l'élaboration du plan d'actions de réduction des risques assorti du catalogue des paramètres de sécurité :

- Les risques majeurs sont identifiés parmi les risques non acceptables sur la base de la valeur maximale des risques moyens. Le bilan financier basé sur les pertes et efforts peut contribuer à la priorisation des actions par les dirigeants.

In fine, chaque action de réduction des risques et paramètre de sécurité, validés par les dirigeants, entrent dans un programme d'actions Sécurité/Qualité des Soins et font l'objet de fiches projets avec suivi.

\subsubsection{Les résultats de l'étude}

\subsubsection{L'AGR système}

\section{La description du système :}

Selon la méthode RELIASEP, le système étudié s'est décomposé de la manière suivante et chacune des phases a fait l'objet de l'analyse.

Encourager le patient à rédiger ses directives anticipées :

- Mettre en œuvre le devoir général d'information du patient sur ses droits en matière de directives anticipées;

- $\quad$ Elaborer un support écrit d'information du patient sur ses droits en matière de directives anticipées ;

- $\quad$ Remettre au patient le support écrit d'information ;

- Donner au patient des informations et explications orales sur ses droits en matière de directives anticipées. 


\section{Parler de management et de gestion éthique. Un exemple : le recueil des}

directives anticipées

- Identifier chaque patient éligible à la rédaction des directives anticipées

- $\quad$ Evaluer la capacité du patient à exprimer librement sa volonté.

- Donner des informations médicales au patient quant à son état de santé

- Donner des informations et des explications médicales au patient sur son état de santé;

- Vérifier la compréhension du patient.

- Recueillir la décision du patient de rédiger ou non ses directives anticipées

- Enregistrer les données dans le dossier informatisé du patient

- $\quad$ Enregistrer l'acte d'information du patient sur ses droits en matière de directives anticipées ;

- $\quad$ Enregistrer l'acte d'information médicale du patient sur son état de santé ;

- Enregistrer les résultats de l'évaluation du patient quant à sa capacité à exprimer librement sa volonté ;

- $\quad$ Enregistrer la décision du patient de rédiger ou non ses directives anticipées.

\section{La cartographie des dangers :}

Au total, 103 éléments ou évènements dangereux ont été identifiés par le groupe de travail :

- 47,57 \% (N = 49) dangers externes au système étudié : politique extérieure, insécurité externe, environnement externe, image externe de l'établissement et client/patient/ usager.

- 29,13\% ( N = 30) dangers liés à la gouvernance du système étudié : culture d'établissement, management, stratégie, communication, éthique, danger juridique et financier.

- 14,56 \% ( $\mathrm{N}=15)$ dangers liés aux études et productions du système étudié : danger opérationnel, danger lié au facteur humain et aux professionnels.

- 8,74 \% (N = 9) dangers liés aux moyens du système étudié : infrastructure, matériels, équipements et système d'information.

\section{La cartographie des situations dangereuses :}

Au total, 311 situations dangereuses ont été identifiées par le groupe de travail. Leur distribution a montré les résultats suivants :

- $55 \%$ des dangers provenaient, par ordre décroissant: des clients/patients/usagers, du management interne à l'établissement et de la politique extérieure à l'établissement. - La phase du système « Mettre en œuvre le devoir général d'information du patient sur ses droits en matière de directives anticipées » était celle qui présentait le plus de vulnérabilité en nombre de situations dangereuses.

Sur le principe de ne retenir que les situations dangereuses résultant d'une interaction forte à très forte et sur la capacité et la volonté de traitement par les dirigeants, seules 101 situations dangereuses ont été retenues pour analyse dans le cadre de l'AGR scénarios, soit 32,47 \% de l'ensemble des situations dangereuses.

Leur distribution a montré les mêmes résultats que précédemment. 


\subsubsection{L'AGR scénarios}

\section{Les paramètres d'évaluation :}

L'échelle de gravité des conséquences:

\begin{tabular}{|c|c|c|c|}
\hline $\begin{array}{c}\text { Classe } \\
\text { de } \\
\text { gravité }\end{array}$ & $\begin{array}{c}\text { Intitulé de la } \\
\text { classe }\end{array}$ & Index & Intitulé des conséquences \\
\hline G1 & Mineure & 10 & Aucun impact sur les performances et la sécurité de l'activité \\
\hline \multirow[t]{5}{*}{ G2 } & \multirow[t]{5}{*}{ Significatif } & 20 & $\begin{array}{l}\text { Dégradation des performances du système sans impact sur } \\
\text { la sécurité }\end{array}$ \\
\hline & & 21 & Insatisfaction du patient ou de son entourage \\
\hline & & 22 & Dégradation de la confiance du patient envers l'équipe \\
\hline & & 23 & $\begin{array}{l}\text { Report dans le temps de la décision du patient de rédiger } \\
\text { ou non ses directives anticipées sans conséquence dans sa } \\
\text { prise en charge }\end{array}$ \\
\hline & & 24 & Insatisfaction du personnel \\
\hline \multirow[t]{6}{*}{ G3 } & \multirow[t]{6}{*}{ Grave } & 30 & $\begin{array}{l}\text { Forte dégradation ou échec des performances du système } \\
\text { sans impact sur la sécurité }\end{array}$ \\
\hline & & 31 & Recours à un médiateur \\
\hline & & 32 & Perte de confiance du patient ou de son entourage \\
\hline & & 33 & $\begin{array}{l}\text { Absence de décision du patient de rédiger ou non ses direc- } \\
\text { tives anticipées sans conséquence dans sa prise en charge }\end{array}$ \\
\hline & & 34 & Turn over de l'équipe \\
\hline & & 35 & $\begin{array}{l}\text { Décision du patient de rédiger ou non ses directives anticipées } \\
\text { non conforme à sa volonté réelle mais sans conséquence dans } \\
\text { sa prise en charge }\end{array}$ \\
\hline \multirow[t]{6}{*}{ G4 } & \multirow[t]{6}{*}{ Critique } & 40 & Dégradation de la sécurité ou de l'intégrité du système \\
\hline & & 41 & Indemnisation à l'amiable \\
\hline & & 42 & $\begin{array}{l}\text { Report dans le temps de la décision du patient de rédiger } \\
\text { ou non ses directives anticipées avec conséquence dans sa } \\
\text { prise en charge }\end{array}$ \\
\hline & & 43 & Prise en compte partielle de la volonté du patient \\
\hline & & 44 & Fuite de la clientèle \\
\hline & & 45 & Directives anticipées non valides \\
\hline \multirow[t]{5}{*}{ G5 } & \multirow[t]{5}{*}{ Catastrophique } & 50 & Forte dégradation ou échec de la sécurité ou perte du système \\
\hline & & 51 & $\begin{array}{l}\text { Indemnisation financière de l'établissement suite à } \\
\text { condamnation }\end{array}$ \\
\hline & & 52 & $\begin{array}{l}\text { Maintien déraisonnable en vie ou fin de vie du patient non } \\
\text { conforme à sa volonté réelle }\end{array}$ \\
\hline & & 53 & Interdiction d'exercice du médecin responsable du patient \\
\hline & & 54 & $\begin{array}{l}\text { Absence de décision du patient de rédiger ou non ses direc- } \\
\text { tives anticipées avec conséquence dans sa prise en charge }\end{array}$ \\
\hline
\end{tabular}




\section{Parler de management et de gestion éthique. Un exemple : le recueil des directives anticipées}

\section{L'échelle de vraisemblance de la gravité des conséquences:}

Pour élaborer l'échelle de vraisemblance et notamment la période de retour, le groupe de travail s'est appuyé sur les données d'activité à temps complet et la durée moyenne de séjour de l'année 2012, tous patients confondus.

\begin{tabular}{|l|l|l|l|}
\hline $\begin{array}{c}\text { Classe de } \\
\text { vraisemblance }\end{array}$ & $\begin{array}{c}\text { Intitulé de la } \\
\text { classe }\end{array}$ & Index & \multicolumn{1}{|c|}{ Intitulé des vraisemblances } \\
\hline V1 & Très improbable & 1 & $\begin{array}{l}\text { Moins d'un patient tous les 550 patients, soit moins } \\
\text { d'une fois par an }\end{array}$ \\
\hline V2 & $\begin{array}{l}\text { Très peu } \\
\text { probable }\end{array}$ & 2 & $\begin{array}{l}\text { Entre un patient tous les 550 patients et un patient } \\
\text { tous les 300 patients, soit entre une fois par an et } \\
\text { une fois tous les 6 mois }\end{array}$ \\
\hline V3 & Peu probable & 3 & $\begin{array}{l}\text { Entre un patient tous les 300 patients et un patient } \\
\text { tous les 150 patients, soit entre une fois tous les 6 } \\
\text { mois et une fois tous les 3 mois }\end{array}$ \\
\hline V4 & Probable & 4 & $\begin{array}{l}\text { Entre un patient tous les 150 patients et un patient } \\
\text { tous les 50 patients, soit entre une fois tous les 3 } \\
\text { mois et une fois par mois }\end{array}$ \\
\hline V5 & $\begin{array}{l}\text { Très probable à } \\
\text { certain }\end{array}$ & 50 & $\begin{array}{l}\text { Plus d'un patient tous les 50 patients, soit plus d'une } \\
\text { une fois par mois }\end{array}$ \\
\hline
\end{tabular}

\section{Les éléments d'acceptation du risque et de décision :}

\section{L'échelle de criticité:}

L'établissement a utilisé celle proposée par l'Ecole Centrale Paris dans le cadre du mastère spécialisé.

\begin{tabular}{|c|l|l|}
\hline $\begin{array}{c}\text { Classe de } \\
\text { criticité }\end{array}$ & \multicolumn{1}{|c|}{$\begin{array}{c}\text { Intitulé de la } \\
\text { classe }\end{array}$} & \multicolumn{1}{c|}{ Conduite à tenir } \\
\hline C1 & Acceptable & Aucune action n'est à entreprendre \\
\hline C2 & $\begin{array}{l}\text { Tolérable sous } \\
\text { contrôle }\end{array}$ & $\begin{array}{l}\text { Un suivi doit être organisé en termes de gestion du risque (y } \\
\text { compris transfert) }\end{array}$ \\
\hline C3 & Non acceptable & $\begin{array}{l}\text { On doit refuser la situation et prendre des mesures en réduc- } \\
\text { tion des risques ou refuser tout ou partie de l'activité }\end{array}$ \\
\hline
\end{tabular}

La matrice de criticité:

\begin{tabular}{|c|c|c|c|c|c|c|}
\hline & & \multicolumn{5}{|c|}{ Gravité des conséquences } \\
\hline & & G1 & G2 & G3 & G4 & G5 \\
\hline \multirow{5}{*}{ 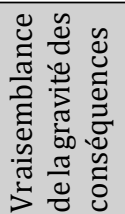 } & V5 & & & & & \\
\hline & V4 & & & & & \\
\hline & V3 & & & & & \\
\hline & V2 & & & & & \\
\hline & V1 & & & & & \\
\hline
\end{tabular}


Les pertes et efforts :

La perte a été calculée au regard de l'estimation des pertes financières consécutives à la gravité des conséquences des scénarios redoutés identifiés. Seules les pertes financières subies par l'établissement ont été prises en compte.

L'effort a été calculé au regard de l'estimation des efforts financiers à fournir par l'établissement s'il devait mettre en œuvre les actions de réduction des risques associées aux scénarios redoutés identifiés.

\begin{tabular}{|l|c|c|c|c|c|c|}
\hline \multirow{2}{*}{ Phases du système étudiées } & \multicolumn{6}{|c|}{ Valeur des pertes et efforts par index (euros) } \\
\cline { 2 - 8 } & \multicolumn{2}{|c|}{ Index des pertes } & \multicolumn{3}{|c|}{ Index des efforts } \\
\cline { 3 - 8 } & P1 & P2 & P3 & E1 & E2 & E3 \\
\hline $\begin{array}{l}\text { Mettre en œuvre le devoir général d'informa- } \\
\text { tion du patient sur ses droits en matière de } \\
\text { directives anticipées }\end{array}$ & 500 & 2.000 & 15.000 & 1.000 & 10.000 & 125.000 \\
\hline $\begin{array}{l}\text { Identifier chaque patient éligible à la rédaction } \\
\text { de directives anticipées }\end{array}$ & 500 & 2.000 & 45.000 & 500 & 8.000 & 80.000 \\
\hline $\begin{array}{l}\text { Donner des informations médicales au patient } \\
\text { sur son état de santé }\end{array}$ & & & 2.000 & 500 & 3.000 & 10.000 \\
\hline $\begin{array}{l}\text { Recueillir la décision du patient de rédiger ou } \\
\text { non ses directives anticipées }\end{array}$ & 500 & 2.000 & 45.000 & 1.000 & 4.000 & 8.000 \\
\hline
\end{tabular}

\section{La cartographie des risques :}

Au total, 332 scénarios ont été identifiés par le groupe de travail à partir des 101 situations dangereuses retenues précédemment.

\section{Diagnostic initial :}

La distribution des scénarios par criticité initiale a montré les résultats suivants :
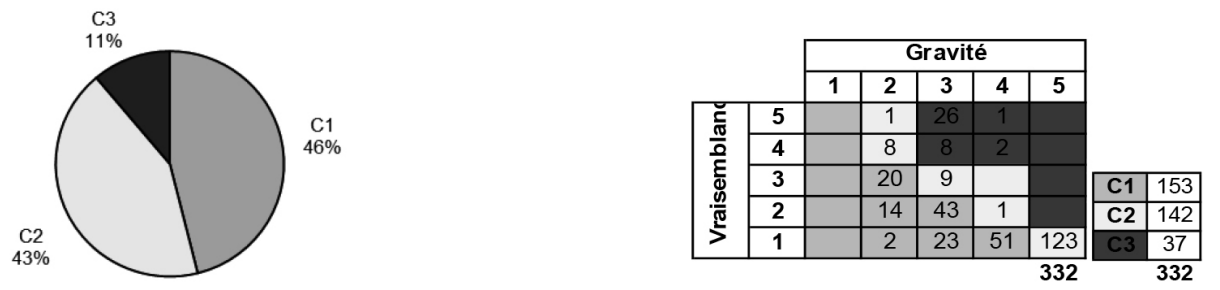

- $11 \%$ des scénarios $(\mathrm{N}=37)$ étaient associés à un risque non acceptable (C3) nécessitant la définition, mise en œuvre et consolidation d'actions de réduction des risques ;

- $43 \%$ des scénarios $(\mathrm{N}=142)$ étaient associés à un risque tolérable sous contrôle (C2) pouvant faire l'objet d'actions de réduction des risques ou être associés à une procédure de gestion de suivi et de contrôle pendant toute la durée de l'activité;

- $100 \%$ des scénarios avaient des conséquences (G2+G3+G4+G5) ;

- 52,71 \% des scénarios portaient atteinte à la sécurité (G4+G5) et 47,29\% à la performance (G2+G3) ; 


\section{Parler de management et de gestion éthique. Un exemple : le recueil des directives anticipées}

- 69,88\% des scénarios avaient des conséquences irréversibles (G3+G5) ;

- Les gravités des conséquences les plus fréquentes correspondaient, premièrement, à une forte dégradation ou un échec de la sécurité ou une perte du système (G5, 37 \%), et, deuxièmement, à une forte dégradation ou un échec des performances du système sans impact sur la sécurité (G3, 32,83\%);

- La vraisemblance de la gravité des conséquences la plus fréquente était celle correspondant à moins d'un patient tous les 550 patients, soit moins d'une fois par an (V1, 59,94 \%) ;

- 70,27 \% des scénarios associés à un risque non acceptable (C3) avaient des conséquences irréversibles sans impact sur la sécurité (G3) et une vraisemblance de survenue de ces conséquences de l'ordre de plus d'une fois par mois (V5);

- 86,62 \% des scénarios associés à un risque tolérable sous contrôle (C2) avaient des conséquences irréversibles avec impact sur la sécurité (G5) et une vraisemblance de survenue de ces conséquences de l'ordre de moins d'une fois par an (V1).

La distribution des risques moyens cumulés par niveau de criticité initiale a montré les résultats suivants :

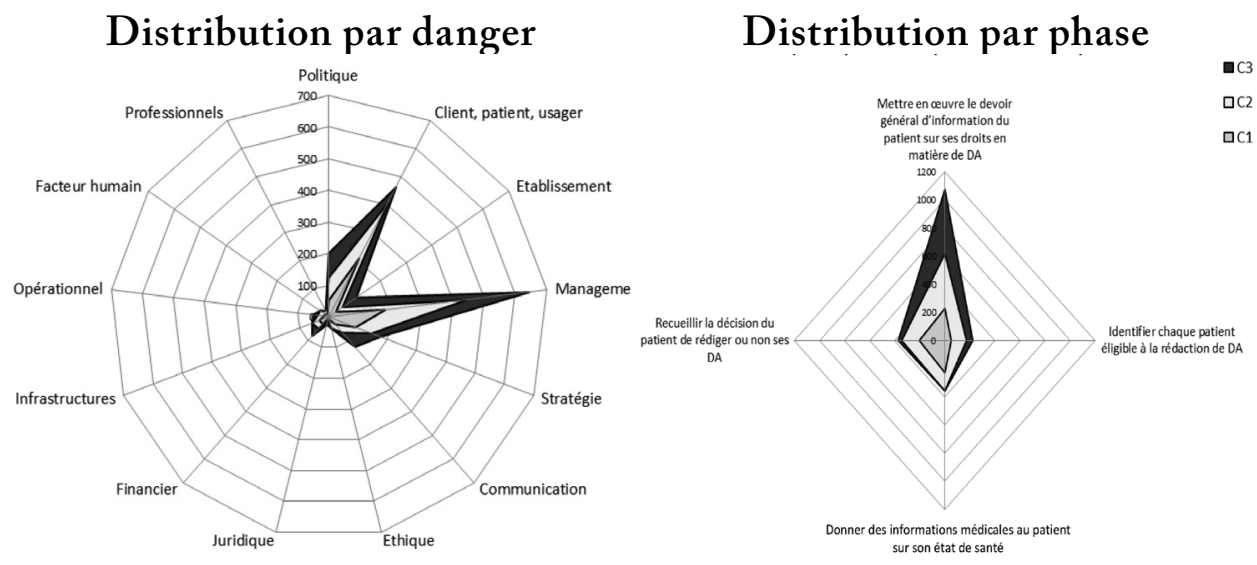

Les deux dangers : Management et Client/Patient/Usager, étaient ceux qui présentaient le plus de potentiel de préjudice au vu du nombre de situations dangereuses et de scénarios générés.

La distribution des risques moyens cumulés par niveau de criticité initiale et par danger montrait également la prédominance de ces deux dangers, quel que soit le niveau de criticité initial pris en compte. Elle permettait de conclure que les efforts à fournir pour traiter les risques devraient se concentrer sur ces deux dangers prioritairement.

La phase du système consistant à mettre en œuvre le devoir général d'information du patient sur ses droits en matière de directives anticipées était la plus vulnérable en termes de nombre de situations dangereuses et de scénarios associés. La distribution des risques moyens cumulés par niveau de criticité initiale et par phase, permettait également de conclure que les efforts de traitement des risques devraient être concentrés sur cette phase. 
Concernant le coût des risques :

- $76 \%$ reposaient, pratiquement à parts égales, sur la phase de mise en œuvre du devoir général d'information du patient sur ses droits en matière de directives anticipées et celle du recueil de la décision du patient de rédiger ou non ses directives anticipées. Ces chiffres, rapportés au nombre de scénarios à traiter, montraient néanmoins que la perte moyenne d'un scénario de la deuxième phase était 3,26 fois plus élevée que celle d'un scénario de la première phase.

- Du point de vue des conséquences, «le maintien déraisonnable en vie ou une fin de vie du patient non conforme à sa volonté réelle », cumulait, à elle seule, $54 \%$ des pertes financières. Cette proportion élevée s'expliquait par le nombre de scénarios concernés et non par le coût individuel de cette conséquence. En effet, l'assurance de l'établissement couvrait ce risque.

Traitement envisagé de l'ensemble des risques :

Au total, 33 actions de réduction des risques ont été identifiées en traitement des 179 scénarios qui le nécessitaient ( $\mathrm{C} 3$ et $\mathrm{C} 2$ ).

En complément, 10 paramètres de sécurité ont été définis à partir des 110 scénarios au risque résiduel de criticité 2 .

Quatre actions sont arrivées en tête au regard du nombre de scénarios couverts :

- Elaborer un programme de Développement Professionnel Continu (DPC), obligatoire pour les professionnels du pôle et ouvert aux partenaires médicaux de proximité ;

- Réactualiser la procédure relative à la prise en charge en Soins de Suite, de Réadaptation et de Médecine Physique ;

- $\quad$ Soumettre le support écrit d'information du patient sur ses droits en matière de directives anticipées au Réseau Champ Ardennais Accompagnement Soins Palliatifs (RécaP) ;

- Intégrer au programme d'amélioration de la Qualité et de la Sécurité des Soins (paQSS), un objectif opérationnel sur l'encouragement des patients à rédiger leurs directives anticipées.

Les deux dangers : Management et Client/Patient/Usager, les deux phases : Mettre en œuvre le devoir général d'information du patient sur ses droits en matière de directives anticipées et recueillir la décision du patient de rédiger ou non ses directives anticipées et enfin le couple: Management/Information du patient sur ses droits en matière de directives anticipées, étaient ceux qui nécessitaient le plus de traitement.

Le diagramme de FARMER a mis en évidence, en comparant la répartition des risques initiaux moyens par danger à celle des risques résiduels, que les actions de réduction des risques étaient du domaine de la protection et de la prévention avec une préférence pour cette dernière.

La même analyse par phase a également montré l'efficacité d'actions mixtes, permettant de gagner en termes de gravité et de vraisemblance. C'est l'information du patient sur ses droits en matière de directives anticipées qui aurait le mieux progressé. Toutes les phases seraient passées en zone d'acceptabilité. Seule la phase de recueil de la 
Parler de management et de gestion éthique. Un exemple : le recueil des directives anticipées

décision du patient de rédiger ou non ses directives anticipées aurait été maintenue à la jonction $\mathrm{C} 1 / \mathrm{C} 2$.

\section{Diagramme de FARMER par phase sans traitement des risques}

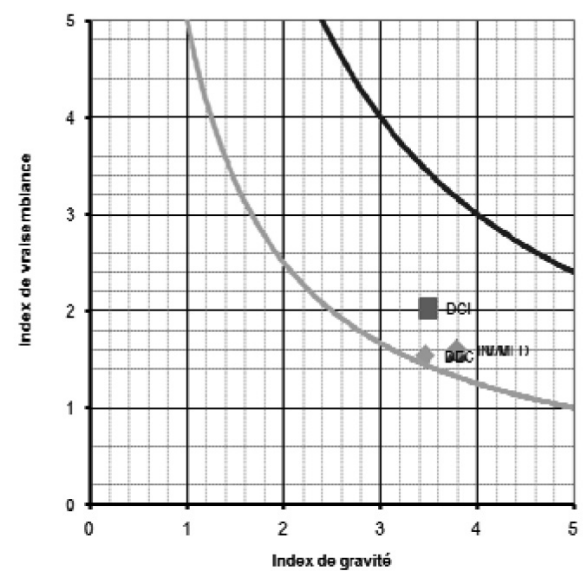

Diagramme de FARMER par phase si mise en place de l'ensemble des actions envisagées

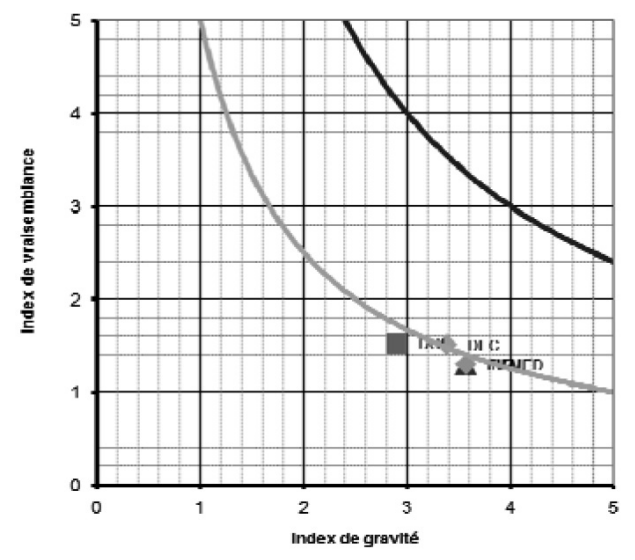

Concernant le coût du traitement des risques :

- Du point de vue des différentes phases du système, 67,4 \% des efforts financiers à fournir pour traiter les risques auraient été liés à la phase de mise en œuvre du devoir général d'information du patient sur ses droits en matière de directives anticipées.

- Du point de vue des conséquences : 33 \% des efforts financiers auraient été liés au maintien déraisonnable en vie ou une fin de vie non conforme à la volonté réelle du patient. Cela aurait représenté un coût moyen de traitement par scénario d'environ 13.700 euros. À noter également que 22,1 \% des efforts financiers auraient été associés au traitement des risques ayant pour conséquence une décision du patient de rédiger ou non ses directives anticipées, non conforme à sa volonté réelle, mais sans conséquence dans sa prise en charge. Ceci aurait représenté un coût moyen de traitement par scénario d'environ 12.900 euros. À noter aussi que 16,9\% des efforts financiers auraient été associés au traitement des risques ayant pour conséquence l'absence de décision du patient de rédiger ou non ses directives anticipées avec conséquence dans sa prise en charge. Ceci aurait représenté un coût moyen de traitement par scénario d'environ 11.000 euros. À noter enfin que 12,7 \% des efforts financiers auraient été associés au traitement des risques ayant pour conséquence l'absence de décision du patient de rédiger ou non ses directives anticipées sans conséquence dans sa prise en charge. Ceci aurait représenté un coût moyen de traitement par scénario d'environ 7.900 euros.

Du point de vue des phases du système, le bilan global effort/perte n'était pas en faveur du traitement des risques en affichant un coût de traitement 5,53 fois supérieur au coût des risques sans traitement. Dans le meilleur des cas, l'opération financière était 
nulle et cela concernait la phase de recueil de la décision du patient de rédiger ou non ses directives anticipées.

Du point de vue des conséquences, le bilan financier global était nul. Par contre, il était en faveur du traitement des risques associés aux conséquences de gravité 5 . Il en était de même pour le traitement de la conséquence : « fuite de la clientèle» (G44).

\section{Diagramme effort/perte par phase}

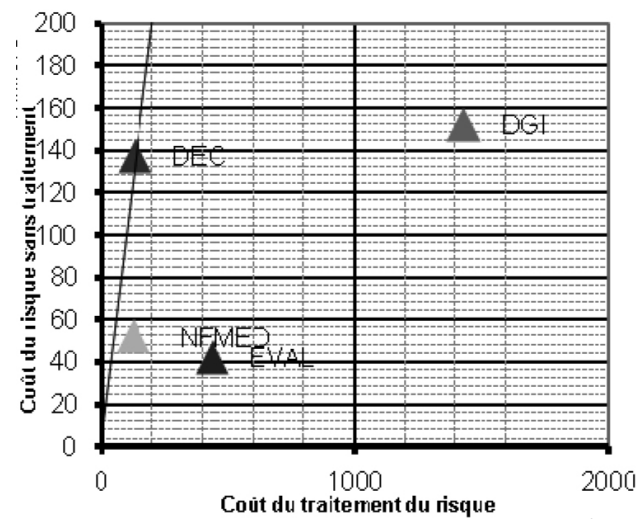

Diagramme effort/perte par conséquence

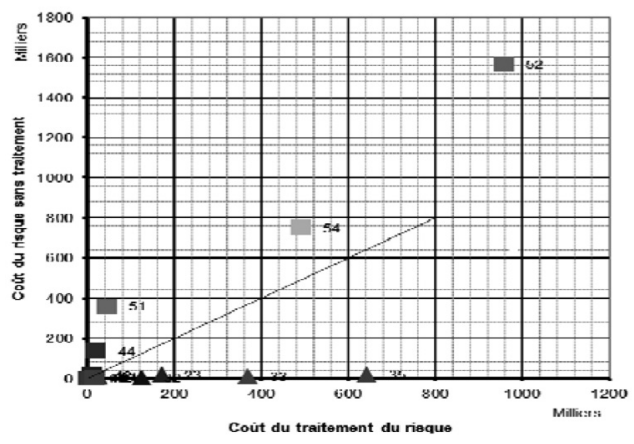

En décidant de mettre en œuvre l'ensemble des actions de réduction des risques identifiées, la répartition des scénarios par criticité résiduelle aurait pris les allures suivantes :
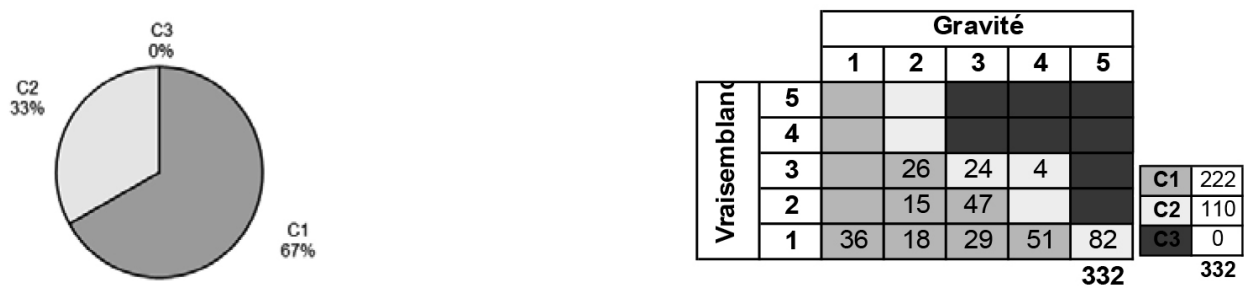

- $\quad$ Il n'y aurait plus eu de scénario associé à un risque non acceptable (C3) ;

- $\quad 33 \%$ des scénarios $(\mathrm{N}=110)$ auraient désormais été qualifiés de tolérables sous contrôle (C2) ;

- $\quad$ On aurait réduit de 38,88 \% le nombre initial des scénarios à traiter ou à surveiller (C3+C2) et augmenter de 45,65\% celui des scénarios acceptables en l'état (C1) ; - On aurait gagné en termes de sécurité et de réversibilité des conséquences en réduisant de $23 \%$ le nombre de scénarios aux conséquences avec impact sur la sécurité (G4+G5) et de 21,55 \% le nombre de scénarios aux conséquences irréversibles $(\mathrm{G} 3+\mathrm{G} 5)$;

- Tous les scénarios auraient été associés à une vraisemblance de la gravité des conséquences correspondant au plus à un patient tous les 150 patients, soit au plus à une fois tous les trois mois ;

- $\quad$ Les scénarios associés à un risque tolérable sous contrôle (C2) ayant des conséquences irréversibles avec impact sur la sécurité (G5) et une vraisemblance de survenue de ces conséquences de l'ordre de moins d'une fois par an (V1), auraient toujours été les plus fréquents malgré une baisse de 33,33\%. 


\subsubsection{L'identification des risques majeurs et l'élaboration du plan d'actions de réduction des risques assorti du catalogue des paramètres de sécurité}

Les risques majeurs appartiennent aux risques non acceptables et s'identifient sur la base des valeurs maximales des risques moyens.

Trois risques majeurs ont été identifiés, de risques moyens 20 et 16.

L'établissement a également souhaité examiner les risques non acceptables de valeur 15 , considérant qu'il retiendrait ceux ayant des conséquences en termes de sécurité. Mais aucun ne correspondait à ce critère.

\section{Risque majeur identifié de risque moyen 20 :}

\begin{tabular}{|c|c|c|c|}
\hline $\begin{array}{l}\text { Risque } \\
\text { moyen }\end{array}$ & $\begin{array}{c}\text { PHASE } \\
\text { Sous-phase du système }\end{array}$ & $\begin{array}{c}\text { Danger générique } \\
\text { Danger spécifique } \\
\text { Elément ou évènement } \\
\text { dangereux }\end{array}$ & $\begin{array}{l}\text { Action de réduction des } \\
\text { risques associés +/- pa- } \\
\text { ramètre de sécurité }\end{array}$ \\
\hline 20 & $\begin{array}{l}\text { EVAL } \\
\text { Identifier chaque patient } \\
\text { éligible à la rédaction de } \\
\text { directives anticipées }\end{array}$ & $\begin{array}{l}\text { Politique } \\
\text { National } \\
\text { Loi imprécise }\end{array}$ & $\begin{array}{l}\text { Action : A14 } \\
\text { Elaborer un programme } \\
\text { de DPC sur le recueil des } \\
\text { directives anticipées, obli- } \\
\text { gatoire pour les profes- } \\
\text { sionnels du pôle et ouvert } \\
\text { aux partenaires médicaux } \\
\text { de proximité } \\
\text { Paramètre de sécurité } \\
\text { associé : P08 } \\
\text { Suivre le programme }\end{array}$ \\
\hline
\end{tabular}

Le rapport perte/effort correspondant à ce scénario n'était pas en faveur d'un traitement du risque (perte en l'absence de traitement estimée à 2.000 euros et coût estimé du traitement : 8.000 euros).

Pour autant, l'action A14 permettait de traiter 32,43\% de l'ensemble des scénarios aux risques de criticité initiale 3 et $26 \%$ de l'ensemble des scénarios aux risques de criticité initiale 2.

Ainsi, les pertes estimées en l'absence de traitement des risques étaient de 132.000 euros et le bilan financier global devenait alors économiquement très rentable. 
Risques majeurs identifiés de risque moyen 16 :

\begin{tabular}{|c|c|c|c|}
\hline Risque moyen & $\begin{array}{c}\text { PHASE } \\
\text { Sous-phase du système }\end{array}$ & $\begin{array}{c}\text { Danger générique } \\
\text { Danger spécifique } \\
\text { Elément ou évènement } \\
\text { dangereux }\end{array}$ & $\begin{array}{l}\text { Action de réduction des } \\
\text { risques associés }+/- \text { pa- } \\
\text { ramètre de sécurité }\end{array}$ \\
\hline 16 & $\begin{array}{l}\text { EVAL } \\
\text { Identifier chaque } \\
\text { patient éligible à la } \\
\text { rédaction de directives } \\
\text { anticipées }\end{array}$ & $\begin{array}{l}\text { Management } \\
\text { Organisation } \\
\text { Absence de retour d'ex- } \\
\text { périence avec équipe } \\
\text { concernée suite à un } \\
\text { évènement indésirable } \\
\text { en lien avec l'identifica- } \\
\text { tion des patients éli- } \\
\text { gibles à la rédaction de } \\
\text { directives anticipées }\end{array}$ & \multirow{2}{*}{$\begin{array}{l}\text { Action : A10 } \\
\text { Former } 5 \text { profession- } \\
\text { nels à l'analyse des } \\
\text { causes profondes } \\
\text { des évènements } \\
\text { indésirables } \\
\\
\text { Paramètre de sécurité } \\
\text { associé : P03 } \\
\text { Suivre les évènements } \\
\text { indésirables sur le pôle }\end{array}$} \\
\hline 16 & $\begin{array}{l}\text { DEC } \\
\text { Recueillir la décision } \\
\text { du patient de rédiger } \\
\text { ou non ses directives } \\
\text { anticipées }\end{array}$ & \begin{tabular}{|l|} 
Management \\
Organisation \\
Absence de retour \\
d'expérience avec \\
équipe concernée suite \\
à un évènement indé- \\
sirable en lien avec le \\
recueil de la décision \\
du patient de rédiger \\
ou non ses directives \\
anticipées
\end{tabular} & \\
\hline
\end{tabular}

Le rapport perte/effort correspondant à ce scénario était d'emblée favorable (perte en l'absence de traitement estimée à 47.000 euros et coût estimé du traitement: 8.000 euros).

Ces deux actions ainsi que leur paramètre de sécurité associé ont été validées par la direction et ont intégré le programme d'amélioration continue de la qualité et de la sécurité des soins de l'établissement.

\subsection{Le management éthique comme forme d'innovation managériale à l'hôpital}

La méthode d'analyse globale des risques appliquée au recueil des directives anticipées s'est révélée particulièrement efficace. Elle a permis de remplacer une approche intuitive, pressentie sur un tel sujet, par une méthode rigoureuse identifiant les risques majeurs encourus par l'établissement et les patients et de proposer des actions de réduction des risques pertinentes, tant du point de vue de leur efficacité pressentie que de leur impact économique.

Attention pour autant. Tous les risques non acceptables identifiés en début d'étude, c'est-à-dire des risques que l'on devait refuser et pour lesquels on aurait du prendre des mesures en réduction des risques ou refuser tout ou partie de l'activité, n'ont pas été traités. Est-ce à dire que l'on finirait par les accepter et remettre en cause la matrice 


\section{Parler de management et de gestion éthique. Un exemple : le recueil des directives anticipées}

de criticité, outil managérial de décision? La question reste entière. Rappelons cependant qu'ils n'avaient aucune conséquence en termes de sécurité. In fine, l'établissement a donc accepté, a posteriori, des risques aux conséquences sur la performance qu'il n'acceptait pas initialement.

Dépassant le simple périmètre du pôle, voire de l'établissement, la méthode a conduit à identifier puis valider une action ouverte aux partenaires de l'établissement: hôpital, ville, médico-social qui devrait permettre de renforcer le travail en réseau de proximité (travail de concertation et de coordination avec l'extérieur, comme fondements d'un management participatif et ouvert). Elle a également permis de fédérer les acteurs du pôle autour d'une démarche structurée devenant structurante pour leur projet de recueil des directives anticipées (démarche de structuration de la réflexion éthique comme base d'un projet managérial collectif). Enfin, cette étude a mis en lumière toute l'étendue possible de la gestion des risques en établissement et réseaux de santé, au-delà des domaines qui lui sont communément attribués.

Cette étude montre donc qu'il est possible de concevoir les valeurs éthiques portées par les droits des patients, comme sources d'innovations managériales à l'hôpital. Comme le souligne Birkensshaw et al. (2008), l'innovation managériale est « ce qui modifie substantiellement la façon dont les tâches de management sont effectuées et/ou les structures traditionnelles de l'organisation lui permettent ainsi de mieux atteindre ses objectifs ». La réalisation de l'étude en soi a modifié l'organisation des équipes soignantes, notamment dans la collecte du recueil des directives anticipées et le dialogue avec les patients sur ce sujet. L'innovation managériale, posée par cette nouvelle approche des droits des patients dans le domaine de la fin de vie, prend forme sous de nouveaux dispositifs de management (avec notamment la création d'un comité de pilotage pluri professionnel), de nouveaux outils de gestions (l'analyse globale des risques), et d'autre part, elle peut se traduire par de nouvelles formes organisationnelles transformant les structures (intégration de cette étude au niveau régional) (Nobre, 2013).

Hatchuel et Weil (1992) ont montré qu'une technique managériale (IOC) se définissait par trois éléments en interaction : un substrat technique, une philosophie gestionnaire et une vision simplifiée des relations organisationnelles. Ici le substrat technique correspond à cette méthode d'analyse des risques : «il s'agit du système de concepts qui désigne les objets et les objectifs formant les cibles d'une rationalisation ». Cette philosophie « dite gestionnaire » induite par cette étude, correspond à deux logiques l'une d'amélioration du fonctionnement de l'organisation et la seconde à une logique de résolution de problèmes complexes par des relations entre les acteurs (avec l'application des droits des patients par les professionnels de santé et les gestionnaires de l'établissement) et non par une modélisation abstraite. Le degré d'innovation managériale est important, dans la mesure où rien n'était mis en place dans ce domaine, la distance entre l'innovation et l'organisation est grande, le degré de contextualisation interne de l'innovation est faible. Comme l'écrit Albert David : "Plus la distance qui existe entre l'innovation et l'organisation qui l'adopte est grande, plus le degré de contextualisation interne est élevé. Qualitativement, cette distance correspond non seulement à l'écart entre le fonctionnement présent et ce que l'on imagine du fonctionnement futur, mais aussi à la longueur et à la difficulté du chemin à parcourir pour que l'innovation fonctionne dans l'organisation » (Albert David, 1996). 
Pouvoir intégrer cette innovation managériale au fonctionnement de l'établissement implique son adaptation au modèle politique mis en place.

Adossée à une philosophie gestionnaire, nous défendons l'hypothèse ici que cet exemple met en exergue un modèle politique de l'établissement. Ce modèle fonctionne en trois moments (Albert David, 1996). Le premier moment d'énoncé des cadrages (la mise en place des scénarios), de diffusion dans les esprits (la présentation aux instances de l'établissement) des rôles correspondant aux relations nouvelles (l'approche du patient potentiel en termes de recueil des directives anticipées). C'est une période déstabilisante, l'annonce des changements peut provoquer des tensions entre le modèle présenté et les pratiques de terrain existantes. À ce stade de la philosophie gestionnaire, il faut trouver l'adhésion des acteurs et l'aspect réglementaire de cette étude (application de la loi Léonetti) est un levier mobilisé. Dans une seconde phase, les acteurs s'approprient les cadres méthodologiques proposés. Les nouveaux rôles sont approfondis (par exemple le remaniement de la fiche de poste du psychologue, l'ouverture du poste médical, la reconnaissance du métier de coordonnateur de gestion des soins). La question devient alors la conformité de cette philosophie gestionnaire de l'innovation aux pratiques soignantes.

Une seconde crise peut apparaître, si les acteurs ne souhaitent pas investir ces rôles. La troisième phase est celle qui aboutit à la transformation et l'intégration des rôles des acteurs et des connaissances afférentes notamment en termes d'accompagnement des patients dans l'accès à l'information au recueil des directives anticipées. On évalue la réussite de cette phase à la capacité de ce nouveau système à répondre aux difficultés rencontrées, à la reformulation des problèmes, à la remontée des entretiens avec les patients et au degré de satisfaction ressentis par les uns et les autres (personnel soignant/patients).

\section{Conclusion}

La transcription juridique des droits des patients dans une logique de gestion des risques permet, à partir de résultats issus d'une approche de gestion des risques, de déterminer les bases du management à adopter. L'élaboration d'un projet managérial énonce des valeurs partagées et cette étude permet de les décliner objectivement: rendre compatible les évolutions de l'hôpital avec la démarche éthique, rappeler les valeurs communes du service public hospitalier en les corrélant avec les droits des personnes et les valeurs éthiques (égalité, neutralité, médiation, respect de la réglementation, respect du consentement de la personne.)

Cela nécessite de développer des principes de management éthique participatif où de nouveaux profils de managers (à la fois directeur d'établissement et éthicien ?) seraient appréciés (Bréhaux, 2014). On observe alors un retour théorique de la pensée de Mary Parker Follett, pour qui le manager-jardinier est un créateur des possibles faisant des choix dans un univers mouvant, changeant (Groutel, 2014). Le coordonnateur de la gestion des risques associés aux soins pourrait-il être un nouveau manager créatif? Cela rejoint la réflexion de Mintzberg pour qui le manager est celui qui initie des projets et qui est lanceur d'idées nouvelles (Damart, Kletz, 2014). 


\section{Parler de management et de gestion éthique. Un exemple : le recueil des directives anticipées}

On note une progression «décalée » entre la reconnaissance d'un droit-liberté de rédiger des directives anticipées et sa transcription rapide et concrète en termes de démarche qualité en établissement. Si bien qu'une tension réside entre le libre-choix d'une personne et le caractère contraignant d'une réglementation à une époque donnée.

\section{Bibliographie}

AMAISEN J.-C., LE COZ P. (2008), «Avis numéro 111 sur les problèmes éthiques posés par l'utilisation des cadavres à des fins de conservation ou d'exposition muséale », Cahiers du CCNE, Paris.

AUBRY R. (2013), « Numéro 121 de l'avis du CCNE, fin de vie, autonomie de la personne, volonté de mourir », Cahiers du CCNE.

BREHAUX K. (2014), «Quelle place pour les droits individuels et les libertés publiques? La question de l'euthanasie aujourd'hui en France ", Communication, séminaire de recherche, Cevipof, 15 décembre 2015, à paraître.

CODE CIVIL FRANÇAIS, Article 16, www.legifrance.gouv.fr

CODE DE LA SANTE PUBLIQUE, article 1111-11 du code de la santé publique, www. legifrance.gouv.fr

DESCARTES R. (1997), Troisième Méditation Métaphysique, Editions Garnier Flammarion, Paris.

FOUCAULT M. (1975), Surveiller et punir, Naissance de la prison, Gallimard, Paris.

FREMOND-LEJEUNE C. (2012-2013), Application de la loi LEONETTI relative aux droits des patients en fin de vie: Cartographie et gestion des risques du recueil des directives anticipées dans la phase d'encouragement des patients à les rédiger, Master de Gestion des risques et de la sécurité des établissements et réseaux de santé, Ecole Centrale de Paris.

HONNETH A. (2007), La réification, petit traité de théorie critique, Paris, Gallimard.

LAROUSSE, http://www.larousse.fr/dictionnaires/francais/ directive/25782 ?q=directive\#25658

LOI n ${ }^{\circ}$ 2005-370 du 22 avril 2005 relative aux droits des malades et à la fin de vie, www.legifrance.fr

MAITRE ECKHART (1995), Traité du Détachement, Editions Rivage poche, Paris.

MATTEI J.-F., NISAND I. (2003), Où va l'Humanité ? Essai, Paris.

NIETZSCHE F. (2000), Par-delà bien et mal, Garnier-Flammarion, Paris.

NOBRE T. (2013), «L'innovation managériale à l'hôpital : changer les principes du management pour que rien ne change ?», Revue française de gestion, Paris : Lavoisier, numéro 6, 2013, pp 113-127.

NOZICK R. (1974), Anarchie, État et utopie, Presses Universitaires de France, Paris. 
SAVALL H., ZARDET V. (1987), Maîtriser les coûts les performances cachées, Economica, dernière édition 2010.

SPINOZA B. (1977), Éthique, Paris, Vrin. 